\title{
HOOSC128: \\ A More Robust Local Shape Descriptor
}

\author{
Edgar Roman-Rangel and Stephane Marchand-Maillet \\ CVMLab - University of Geneva, Switzerland \\ \{edgar.romanrangel, stephane.marchand-maillet\}@unige.ch
}

\begin{abstract}
This work introduces a new formulation of the Histogram-ofOrientations Shape-Context (HOOSC) descriptor 9, which has shorter dimensionality and higher degree of scale and rotation invariance with respect to previous formulations. We compare the performance of our proposed formulation in terms of dimensionality, computation time, robustness against scale and rotations transformations, retrieval precision and classification accuracy. Our results show that our approach outperforms previous formulations in all cases. We also propose the use of a normalized $\chi^{2}$ test to compare the robustness of descriptors of different dimensionality against scale and rotations transformations.
\end{abstract}

Keywords: Binary images, local shape descriptor, content-based image retrieval, image classification.

\section{Introduction}

The Histogram-of-Orientations Shape-Context (HOOSC) is a local shape descriptor that is invariant to scale and rotation transformations, and that was proposed to deal with complex shapes that are rich in fine visual details [9]. Namely, the HOOSC descriptor was introduced to deal with binary images depicting syllabic instances of the ancient Maya writing system [9], and it has proven more effective than other shape descriptors like Shape Context [1] [7] and the Network of Adjacent Segments [4]; and than descriptors for gray-scale images than cannot handle properly binary images, e.g., SIFT [6] and HOG [2].

An improved version of HOOSC, that encodes only the most informative section around the location of interest, was proposed to improve retrieval performance [10]. Although this second version achieved better retrieval performance of Maya hieroglyphs, there is no guaranty that such a formulation remains optimal for generic shapes that depict object of daily use, e.g., Fig. 1.

In this work, we introduce a new formulation of the HOOSC descriptor that we call HOOSC128. Using a dataset of generic shapes [3], we compare our proposed formulation with the two previous versions of the HOOSC descriptor [9] [10] in terms of dimensionality, computation time, degradation after scale and rotation transformations, retrieval performance, and classification accuracy. Our results show that besides the resulting shorter vector, the proposed HOOSC128 formulation produces local descriptors of higher invariance to scale and rotation 


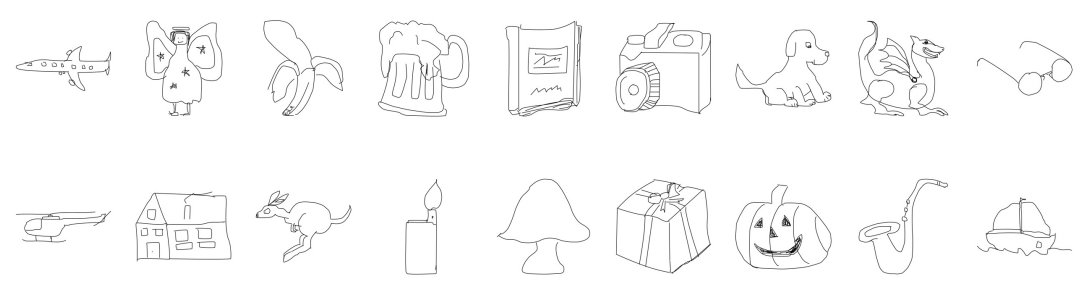

Fig. 1. Some instances of the dataset of generic objects used in this work 3

transformations, which in turn allow to achieve better retrieval and classification performance. Namely, the contributions of this work are:

1. A new formulation of the HOOSC descriptor which has shorter dimensionality and higher discriminative capabilities with respect to previous formulations. It is also more robust against scale and rotation transformations.

2. A thorough comparison of our proposed HOOSC128 definition with respect to two previous formulations. This comparison is both quantitative (dimensionality, computation time, robustness against scale and rotations transformations) and qualitative (retrieval and classification performance).

3. A normalized $\chi^{2}$ test that we propose to compare the robustness of descriptors of different dimensionality after image transformations.

The rest of this paper is organized as follows. Section 2 explains the previous versions of the HOOSC descriptor, and it introduces our proposed HOOSC128 formulation. Section 3 describes the dataset and experimental protocol followed during our experiments. This section also introduces the normalized $\chi^{2}$ test we used to compare descriptors of different dimensionality. Section 4 discusses our results. And section 5 presents our conclusions.

\section{HOOSC Descriptor}

The HOOSC method consists in the local description of a point of interest, which can be selected by a uniform random sampling of all the points in the shape contour [1. Namely, this is a function of the local orientation of its neighbouring points, i.e., the orientation of the contour at the position of such points [9].

The original HOOSC formulation [9], which we refer to as HOOSC0, organizes the set of points around the point of interest by using the same log-polar grid that was first introduced as a support for the Shape Context descriptor [1. This log-polar grid divides the local vicinity around the point of interest into 12 radial intervals uniformly spaced in steps of $\pi / 6$ radians, and 5 distance intervals that are logarithmically spaced from $0.125 T$ to $2 T$, where the parameter $T$ corresponds to the average pairwise distance between all pair of point selected for local description for a given shape. Therefore, the resulting grid contains 60 regions. Fig. 2a shows an example of this log-polar grid. 


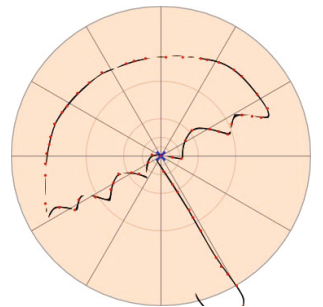

(a) $\operatorname{HOOSC0}$

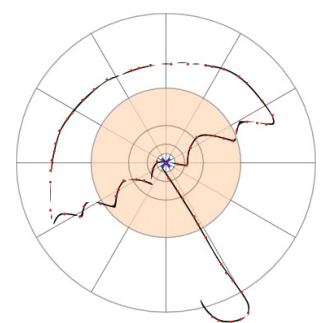

(b) HOOSC4

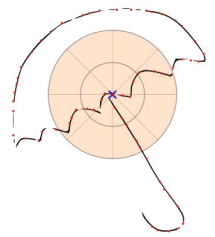

(c) HOOSC128

Fig. 2. Polar grids used by the different HOOSC formulations. The area in orange color correspond to the distance scope that is described by each formulation. HOOSC0 is to the original version of the descriptor 9. HOOSC4 corresponds to an improved version 10, note that the most internal distance interval is in white color as it is not used for description. HOOSC128 is our proposed formulation, note that there are only 2 distance intervals and 8 radial intervals.

After organizing the neighbouring points into the log-polar grid, an 8-bin histogram is built for each of the 60 regions by integrating the local orientation of each of the points within such a region. Such an integration is done using a Gaussian kernel [9]. Finally, all the 60 8-bin histograms are stacked together to build the HOOSC0 descriptor, which therefore, consists in a 480-D vector.

A second formulation of the HOOSC descriptor was proposed to deal with very complex visual structures [10]. Following the naming convention used by Roman-Rangel et al., [10, we refer to this second formulation as HOOSC4. This formulation also relies on the same log-polar grid with the 12 radial intervals. However, instead of using all the 5 distance intervals, it only uses the intervals 2 to 4 , which have shown to be the most informative for very complex structures, i.e., it removes the histograms with higher chance to be all zeros, thus increasing the discriminative potential of the descriptor. Fig $2 \mathrm{~b}$ highlights the distance intervals used by the HOOSC4 formulation.

\section{$2.1 \quad$ HOOSC128}

We noticed that it is beneficial to merge the three most internal distance intervals of the log-polar grid, i.e., the intervals with outer boundaries at $0.125 T, 0.25 T$ and $0.5 T$. There are two reasons for this.

First, the most internal interval is very sparse as it often contains information only about the point of interest [10], as already pointed out. Second, the second and third distance intervals are very local and close to each other, that their respective histograms are often very similar. Based on our observations, we confirmed that the most external distance interval is often empty indeed [10]. Therefore, we propose to use only two distance intervals at $0.5 T$ and $T$. Such wider distance intervals increase the scale invariance potential of the descriptor.

Also, we noticed that 12 radial interval is an excessive splitting of the local context, which harms the potential of the descriptor to be robust enough 
against rotation transformations. Thus, we propose to use only 8 radial intervals uniformly spaced at steps of $\pi / 4$ radians.

The resulting descriptor is a $128-\mathrm{D}$ vector which we call HOOSC128. Fig. 2c shows an example of the resulting polar grid. As shown in section 4, the HOOSC128 allows for better retrieval and classification performance.

Similar to the previous HOOSC formulations, we normalize the HOOSC128 independently per distance interval. Therefore, while HOOSC0 might sum up to 5 and HOOSC4 up to 3, the proposed HOOSC128 might sum up to 2.

\section{Experiments}

This section describes the dataset and the experimental protocol that we used to compare the following three HOOSC formulations:

- HOOSC0: corresponds to the original HOOSC descriptor [9] which uses the log-polar grid of 5 distance intervals and 12 radial intervals.

- HOOSC4: corresponds to the HOOSC descriptor that only uses the most informative section of the distance scope of the log-polar grid, i.e., the second, third, and fourth distance intervals [10, and 12 radial intervals.

- HOOSC128: is our proposed formulation described in section 2.1. This formulation divides the polar grid into 2 distance and 8 radial intervals.

\subsection{Dataset}

The dataset we used to compare the performance of the three HOOSC formulations consists in a collection of 20,000 manual sketches of generic objects [3]. This dataset has 250 visual classes, each of which contains 80 instances.

For experimental purposes, we organized the dataset into 4 disjoint folds of equal size. More precisely, we assigned randomly each of the 80 instances per class to one of the four folds, thus having 20 instances per class in each fold.

Note that the manual sketches in the dataset might have different sizes, however, all of them are centered inside a frame of $1111 \times 1111$ pixels. For efficient experimental performance, we resized all images to $256 \times 256$ pixels.

\subsection{Statistical Validation}

We started by comparing the dimensionality of the three HOOSC formulations, as well as the time they require to compute local descriptors.

After the initial comparison, we also compared their potential for scale and rotation invariance. To this end, we randomly selected 100 points from each class in the dataset, thus 25000 points. For each of these selected points, we computed its three versions of the HOOSC descriptor after applying either a scale or rotation transformation to its source image, as well as the corresponding re-localization of the point of interest in the transformed image. Namely, we used the logarithmic set $\{0.25 x, 0.5 x, 1 x, 2 x, 4 x\}$ as scaling factors, and the set $\left\{0^{\circ}, 30^{\circ}, 60^{\circ}, 90^{\circ}, 120^{\circ}, 150^{\circ}, 180^{\circ}\right\}$ for the rotation angles. 
Note that the re-localization of a point of interest in the transformed images is a straightforward computation of the form,

$$
x^{\prime}=s \times x, \quad y^{\prime}=s \times y,
$$

where $x$ and $y$ denote the coordinates of the point of interest in the source image, $s$ is the scaling factor, and $x^{\prime}$ and $y^{\prime}$ correspond to the coordinates of the point in the scaled image. Likewise, the re-localization of the points of interest in the rotated images is obtained by,

$$
x^{\prime}=x \cos \theta-y \sin \theta, \quad y^{\prime}=x \sin \theta+y \cos \theta,
$$

where $\theta$ indicates the angle of rotation.

After computing the HOOSC descriptors from the transformed images, we compared them with their corresponding descriptors computed from the original image. This comparison was done using the $\chi^{2}$ test statistic, which statistically indicates the degree in which the observed frequency in each dimension of the HOOSC vector is preserved after the transformation. More precisely, we used the following normalized $\chi^{2}$ metric as a measure of distance,

$$
\chi^{2}(\mathbf{o}, \mathbf{t})=\frac{1}{|\mathbf{o}|} \cdot \sum_{i=1}^{|\mathbf{o}|} \frac{\left(o_{i}-t_{i}\right)^{2}}{o_{i}+t_{i}},
$$

where, o and $\mathbf{t}$ denote two HOOSC vectors computed, at the same point of interest, from the original and the transformed images respectively, and $|\cdot|$ is the cardinality operator indicating the size of the vector. Note the use of the denominator $|\mathbf{o}|$ as a normalization parameter. This normalization is required since the simple summation would result in values of different order of magnitude for descriptors of different dimensionality, and therefore hinder their comparison.

\subsection{Retrieval and Classification Performance}

We also report the retrieval and classification performance achieved by each of the three HOOSC formulations. For this purpose, we represented each shape using the Bag-of-Visual-Words (BoW) approach [8] [12. More precisely, we estimated visual vocabularies of 1000 visual words for each HOOSC formulation by using the k-means clustering algorithm [5]. Then, we quantized each local descriptor into one of the visual words in the corresponding vocabulary.

For the retrieval performance case, we report the mean average precision of the first 30 ranked elements (mAP@30), as well as the curves of the average precision versus the standard recall. For the classification performance case, we used the kNN classifier with majority voting, and we report the average classification accuracy as a function of the number of nearest neighbours.

In both cases, retrieval and classification performance evaluations, we relied on a cross-validation approach using the four folds of the data, as explained in section 3.1. We used the L1 norm to compare pairs of BoW representations. 


\section{Results}

This section presents the results of our evaluations. First we compare the dimensionality and computation time of each HOOSC method, followed by a statistical analysis of their scale and rotation invariance capabilities. Then we present the retrieval and classification performance achieved by each method.

\subsection{Dimensionality and Computation Time}

Using each of the three HOOSC formulations, we computed the local descriptors for all 20000 shapes in the dataset and compared the average computation time.

As shown in Table 1 the dimensionality of the proposed HOOSC128 is considerably lower than the dimensionality of the other two formulations, which in turn results in faster computations, i.e., $37.5 \%$ and $16.7 \%$ of improvement with respect to the HOOSC0 and HOOSC4 respectively.

Table 1. Vector size and average time for the three HOOSC formulations

\begin{tabular}{|l|c|c|c|}
\hline Formulation & HOOSC0 & HOOSC4 & HOOSC128 \\
\hline Dimensionality & 480 & 288 & 128 \\
Computation time $(\mathrm{s})$ & $0.08 \pm 0.04$ & $0.06 \pm 0.03$ & $0.05 \pm 0.02$ \\
\hline
\end{tabular}

Table 2 shows the time required by the k-means algorithm [5] to cluster 100 points randomly selected from each class into 1000 clusters. As we can see, the time improves considerably as the dimensionality of the descriptor decreases.

Table 2. Time in seconds required by the k-means clustering algorithm and the three HOOSC formulations to cluster 25000 local descriptors into 1000 clusters

\begin{tabular}{|l|c|c|c|} 
Formulation & HOOSC0 & HOOSC4 & HOOSC128 \\
\hline Time (s) & $3,044.7$ & $1,760.6$ & 703.3
\end{tabular}

\subsection{Scale and Rotation Invariance}

As shown in Fig. 3a, all three HOOSC formulations incur in high levels of degradation when an image is scaled to $25 \%$ of its original size. This degradation is much lower for the scaling factors of $0.5,2$, and 4 . Note that scaling factor of 1 corresponds to a simple copy of the source image, where no degradation happens.

The bars in Fig. 3a also show that the HOOSC0 formulation produces higher degradation rates. The reason for this is the last distance interval, which in this formulation varies a lot after the scale transformation. Although the HOOSC4 formulation varies much less than HOOSC0, it remains less stable than the 


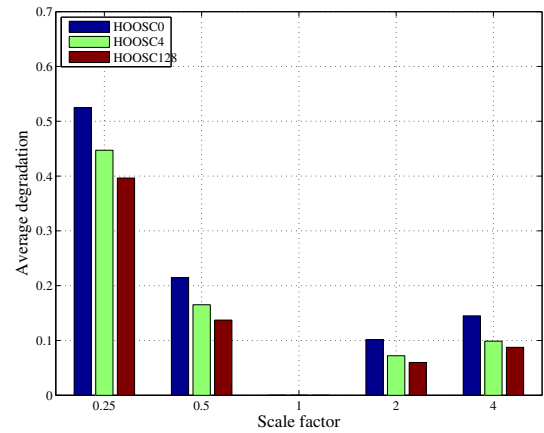

(a) Scale transformation

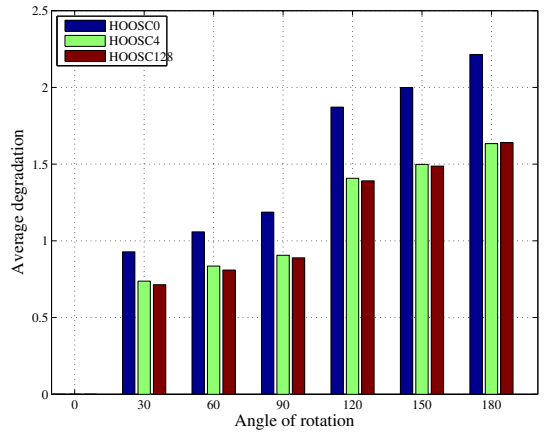

(b) Rotation transformation

Fig. 3. Average degradation after scale and rotation transformations for the three versions of the HOOSC descriptor. The degradation is measured by the $\chi^{2}$ statistic for 25000 points randomly selected from binary images.

proposed HOOSC128 formulation, whose local descriptors are more consistent across scale transformations.

Similar to the degradation rates after scale transformations, we compare the degradation of the local descriptors after rotation transformations in Fig. 3b. In this case, both HOOSC4 and HOOSC128 are far more stable than the original HOOSC0, with HOOSC128 exhibiting a slight higher consistency in all cases.

\subsection{Retrieval Performance}

Besides the statistical comparison of the three HOOSC formulations, we also compared their performance in a retrieval scenario. Table 3 presents the mAP@30 obtained with the three variants of the HOOSC descriptor. As we can see, the proposed HOOSC128 achieves $1.8 \%$ and $3.8 \%$ of improvement in retrieval precision, in absolute terms, with respect to HOOSC0 and HOOSC4 respectively.

Table 3. Mean average precision of the 30 first retrieved elements by each of the three formulations of the HOOSC descriptor

\begin{tabular}{|l|c|c|c|} 
Formulation & HOOSC0 & HOOSC4 & HOOSC128 \\
\hline $\mathrm{mAP} @ 30$ & 0.267 & 0.247 & 0.285
\end{tabular}

Fig. 4 shows the average precision curves as a function of the standard recall rates. Note that in general, HOOSC4 performs lower than HOOSC0, and HOOSC128 improves the performance at all standard intervals. 


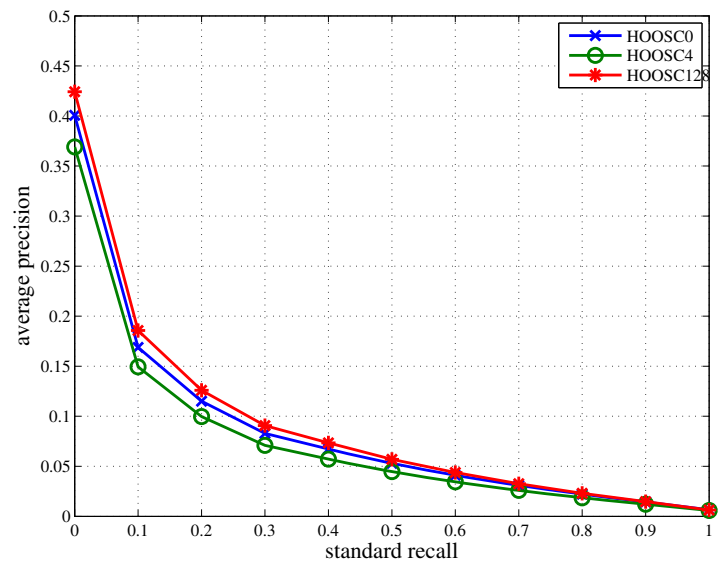

Fig. 4. Retrieval performance: average precision curves versus standard recall rates for the three formulations of the HOOSC descriptor

\subsection{Classification Accuracy}

Fig. 5 shows that the classification performance of three HOOSC formulation remains comparable with the retrieval results. More specifically, HOOSC4 performs the worst in this dataset, whereas HOOSC128 achieves the best classification performance.

In our results, the best performance was achieved using $k=2$ nearest neighbours, which is different from the results reported by Eitz et al., 3], where the best performance for $\mathrm{kNN}$ with hard assignment is obtained with $k=5$. However, note that we have two variations with respect to them: 1) they use SIFT

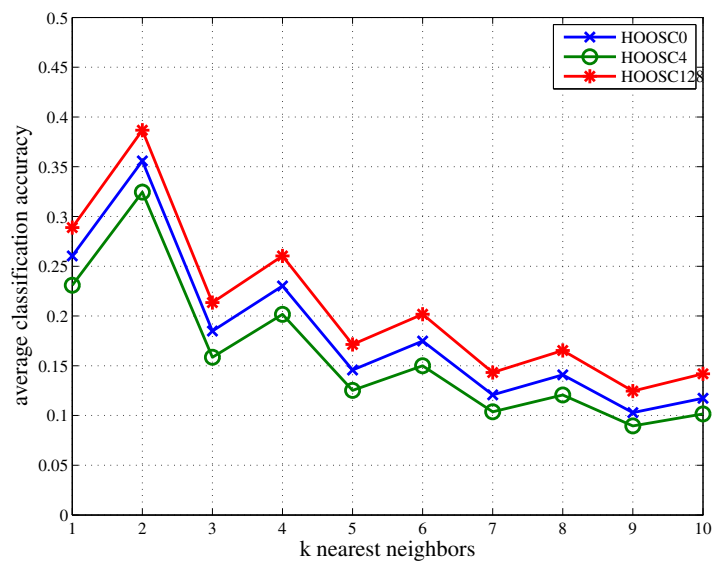

Fig. 5. Classification performance: average classification accuracy for the three formulations of the HOOSC descriptor using the $\mathrm{kNN}$ approach 
descriptors [6] and a vocabulary of 500 visual words; and 2) they used full crossvalidation instead of the 4 -fold cross-validation that we implemented. Moreover, using all the elements in the dataset to train their $\mathrm{kNN}$ classifier, they obtained about $38 \%$ of classification accuracy with kNN and hard assignment (see Fig. 10 in Eitz et al., [3]), whereas we reached $38.67 \%$ with only 20 training elements in each fold, as shown in Fig. [5]

Furthermore, the HOOSC method seems to be a more suitable local descriptor for the binary shapes of the dataset than the well known SIFT descriptor [6], as in Eitz et al., [3, the classification accuracy obtained with 20 training elements is only about $27.5 \%$. This observation is consistent with the intuition that the SIFT descriptor was designed to exploit the local details of intensity images [6], whereas the HOOSC descriptor was tailored to deal with binary images [9].

\subsection{Evaluation on Maya Hieroglyphs}

Finally we evaluated whether the proposed HOOSC128 outperforms HOOSC0 and HOOSC4 retrieving and classifying a set of shapes of the same nature as those for which they were proposed [9] [10]. To this end, we repeated the same experimental protocol used for the generic shapes on a dataset of 6240 Maya syllables equally distributed over 24 classes [11].

Table 4 shows that HOOSC128 also outperforms both HOOSC0 and HOOSC4 in retrieval performance.

Table 4. Mean average precision (mAP) achieved by the three HOOSC formulations on a dataset of Maya hieroglyphs

\begin{tabular}{|c|c|c|c|} 
Formulation & HOOSC0 & HOOSC4 & HOOSC128 \\
\hline mAP & 0.470 & 0.442 & 0.486
\end{tabular}

\section{Conclusions}

We proposed a new formulation of the HOOSC descriptor [9] called HOOSC128, which has smaller dimensionality with respect to previous formulations [9], 10].

We also proposed the use of a normalized $\chi^{2}$ statistic to compare the capabilities of invariance against scale and rotations transformations of three formulations of the HOOSC descriptor that have different dimensionality.

Using a dataset of generic shapes, we also compared the performance of the three HOOSC formulations in the tasks of shape-based image retrieval and image classification. Our results show that the HOOSC128 formulation is more robust to scale and rotation transformations, and it achieves better retrieval and classifications performance than the previous formulations. Similar results were obtained during a retrieval experiment on a dataset of Maya hieroglyphs. 
Acknowledgments. This research was supported by the Secretariat for Education and Research (SEK) under the grant C11.0043, supporting our participation in the COST ACTION MUMIA.

\section{References}

1. Belongie, S., Malik, J., Puzicha, J.: Shape Matching and Object Recognition Using Shape Contexts. IEEE Transactions on Pattern Analysis and Machine Intelligence 24(4), 509-522 (2002)

2. Dalal, N., Triggs, B.: Histograms of Oriented Gradients for Human Detection. In: IEEE Conference on Computer Vision and Pattern Recognition (2005)

3. Eitz, M., Hays, J., Alexa, M.: How Do Humans Sketch Objects? ACM Transactions on Graphics (TOG) - SIGGRAPH 2012 Conference Proc. 31(4), 44 (2012)

4. Ferrari, V., Fevrier, L., Jurie, F., Schmid, C.: Groups of Adjacent Contours for Object Detection. IEEE Transactions on Pattern Analysis and Machine Intelligence 30(1), 36-51 (2008)

5. Lloyd, S.: Least square quantization in PCM. IEEE Transactions on Information Theory 28(2), 129-137 (1982)

6. Lowe, D.: Distinctive Image Features from Scale-Invariant Keypoints. International Journal of Computer Vision 60(2), 91-110 (2004)

7. Mori, G., Belongie, S., Malik, J.: Efficient Shape Matching Using Shape Contexts. IEEE Transactions on Pattern Analysis and Machine Intelligence 27(11), 1832 $1837(2005)$

8. Quelhas, P., Monay, F., Odobez, J.-M., Gatica-Perez, D., Tuytelaars, T.: A Thousand Words in a Scene. IEEE Transactions on Pattern Analysis and Machine Intelligence 29(9), 1575-1589 (2007)

9. Roman-Rangel, E., Pallan, C., Odobez, J.-M., Gatica-Perez, D.: Analyzing Ancient Maya Glyph Collections with Contextual Shape Descriptors. International Journal in Computer Vision, Special Issue in Cultural Heritage and Art Preservation 94(1), 101-117 (2011)

10. Roman-Rangel, E., Pallan, C., Odobez, J.-M., Gatica-Perez, D.: Searching the Past: An Improved Shape Descriptor to Retrieve Maya Hieroglyphs. In: ACM International Conference in Multimedia (2011)

11. Roman-Rangel, E., Marchand-Maillet, S.: Stopwords Detection in Bag-of-VisualWords: The Case of Retrieving Maya Hieroglyphs. In: International Workshop on Multimedia for Cultural Heritage, The International Conference on Image Analysis and Processing (2013)

12. Sivic, J., Zisserman, A.: Video google: A text retrieval approach to object matching in videos. In: IEEE International Conference on Computer Vision (2003) 